\title{
Article
}

\section{Identification of Block-Oriented Nonlinear Processes Using Designed Relay Feedback Tests}

Jyh-Cheng Jeng, Ming-Wei Lee, and Hsiao-Ping Huang

Ind. Eng. Chem. Res., 2005, 44 (7), 2145-2155 • DOI: 10.1021/ie0494840

Downloaded from http://pubs.acs.org on November 18, 2008

\section{More About This Article}

Additional resources and features associated with this article are available within the HTML version:

- $\quad$ Supporting Information

- $\quad$ Access to high resolution figures

- $\quad$ Links to articles and content related to this article

- $\quad$ Copyright permission to reproduce figures and/or text from this article

\section{View the Full Text HTML}

\section{ACS Publications}




\title{
Identification of Block-Oriented Nonlinear Processes Using Designed Relay Feedback Tests
}

\author{
Jyh-Cheng Jeng, ${ }^{\dagger}$ Ming-Wei Lee, ${ }^{\ddagger}$ and Hsiao-Ping Huang*, ${ }^{\dagger}$ \\ Department of Chemical Engineering, National Taiwan University, \\ Taipei, Taiwan 106, Republic of China, and Center for Environmental, Safety and Health Technology \\ Development, Industrial Technology Research Institute, Building 51, 195-10 Section 4, Chung Hsing Road, \\ Chutung, Hsinchu, Taiwan 310, Republic of China
}

A systematic approach to identifying Hammerstein and Wiener models, including the model structure and parameters, for nonlinear processes is presented. A sequence of predesigned relay magnitude changes is applied in a relay feedback experiment to generate dynamic response for identification. By way of this proposed approach, the identifications of linear and nonlinear elements in the block-oriented models are fully separated and the needs for iterative optimization procedures can be relaxed. With the nonlinear models obtained, nonlinear control strategies are developed to achieve a better control performance. The effectiveness of the proposed identification method and control strategy is demonstrated with simulated examples.

\section{Introduction}

Many chemical processes have nonlinear dynamics that can be represented by block-oriented nonlinear models. ${ }^{1}$ These block-oriented models are composed of linear dynamics and nonlinear static functions as blocks in series. The Wiener and Hammerstein models, as shown in Figure 1, are the most popular ones in this class. The Wiener model has a linear dynamic element preceding the nonlinear static element, while the Hammerstein model has a configuration in a reverse order. Because of the increasing popularity and the need for control applications, in past years, a considerable amount of research has been published on the identification of such models. For example, regarding the Weiner process, $\mathrm{Bai}^{2}$ proposed a frequency-domain identification approach by activating the process with a series of sinusoidal inputs that have different frequencies. Vörös ${ }^{3}$ used uniformly distributed random inputs as excitations to the process and a two-segment polynomial for modeling the static nonlinearity. He also presented an iterative method with an internal variable to estimate the model parameters. Sung ${ }^{4}$ used a specially designed test signal consisting of binary and multistep signals to excite a dynamic Hammerstein system and identify the linear dynamic subsystem and nonlinear static function therein. $\mathrm{Bai}^{5}$ adopted the same test signals as those of $\mathrm{Bai}^{2}$ for frequency-domain identification of the Hammerstein process. Notice that the excitations of the process in the aforementioned works are conducted under open loop.

On the other hand, because experiments using relay feedback for excitation can be conducted under closed loop, the autotuning experiments of Åström and Hägglund ${ }^{6}$ have been adopted for the identification of linear and nonlinear Wiener or Hammerstein models. Huang et al. ${ }^{7}$ presented an online adjustable block in a relay feedback loop to identify the static nonlinear part of a

* To whom correspondence should be addressed. Tel.: 8862-2363-8999. Fax: 886-2-2362-3935. E-mail: huanghpc@ ntu.edu.tw.

National Taiwan University.

$\doteqdot$ Industrial Technology Research Institute.

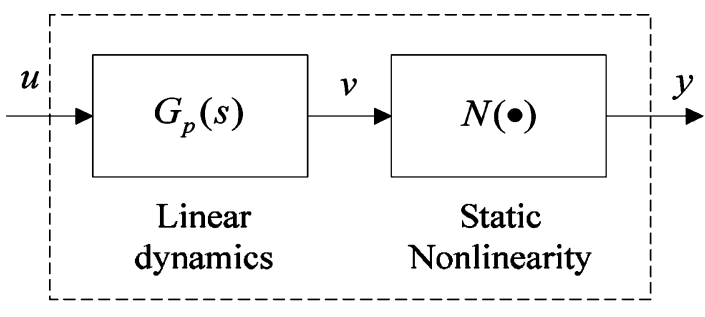

(a)

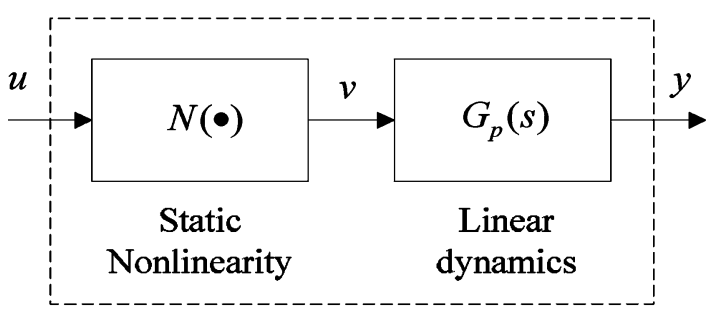

(b)

Figure 1. Two types of block-oriented nonlinear processes: (a) Wiener-type process; (b) Hammerstein-type process.

Wiener model. However, the functional form of this block must be given in advance. Authors such as Luyben and Eskiant ${ }^{8}$ and Balestrino et al. ${ }^{9}$ identified the Hammerstein process by adjusting the relay output to produce symmetrical oscillations. The experiment is conducted in a trial-and-error manner so that the relay feedback experiment has to be repeated with different relays in order to obtain enough equations for identification. Recently, Sung and Lee ${ }^{10}$ proposed a specially designed relay experiment to identify the Wiener process. The relay excitation covers the whole region of the nonlinear function, whereas the information obtained for the linear element is only the ultimate data. Park et al. ${ }^{11}$ also presented a relay feedback approach for the identification of the Hammerstein process, but an additional triangular-type input test is required subsequently.

Most of these methods mentioned assume that the model structure has been determined a priori. There- 
fore, only algorithms for parameter estimation were emphasized. These algorithms are a bit different from each other according to the type of process being identified. Thus, a reasonable procedure for identification is to identify the structure of the model in the first step and choose the appropriate algorithm for parameter estimation in the next.

In this paper, a system of procedures for identifying block-oriented nonlinear models is presented. These procedures include the design of relay experiments and the identification of the model structure and parameters therein. As a first step, the type of model selected is based on an important feature of the response from a relay feedback test. Then, for parameter estimation, a relay feedback experiment is designed accordingly. The static nonlinearity of the proposed model is represented by an invertible function defined on an operation range. After a constant cycle has achieved peak values between the switches of relay, the parameters in this function are determined by a simple least-squares procedure that aims to find coefficients that "best fit" the static nonlinear function to the data obtained from the designed relay experiment. On the basis of the nonlinear function identified, a simple transfer function of the linear subsystem represented by an FOPDT or SOPDT model is determined using the frequency-domain method. By way of this approach, identification of the linear and nonlinear parts is separated in sequence and can be done without any iterative procedure. To demonstrate the advantages of the proposed method, numerical examples and nonlinear control systems based on the identified models have been simulated.

\section{Identification of the Model Structure}

The models in block-oriented form most used for representing the nonlinear processes are the Wiener and Hammerstein types, as shown in Figure 1. Both of them comprise a static nonlinearity $N(\cdot)$ and a linear dynamic subsystem $G_{\mathrm{p}}(s)$, but the order of these components is different. In Figure 1, $u$ and $y$ denote the process input and output, respectively, which are known or measurable, whereas the internal signal, $v$, is not measurable. In this paper, the static nonlinear function is assumed to be monotonic, crossing the origin and, hence, invertible. Besides, the linear dynamic subsystem is assumed to be open-loop stable.

For the modeling of block-oriented nonlinear processes, identification of the model structure is the first step because parameter estimation in the next step counts on the model structure being identified. Several researchers have studied the identification of the model structure, ${ }^{1,12-14}$ but they have different levels of complexity in computation.

In general, it is desirable that experimental data obtained at the stage of structure identification can also be used for parameter estimations so that no extra tests are required. The relay feedback test, as shown in Figure 2a, is suitable for this purpose. The relay output $\left(u_{\mathrm{r}}\right)$ is given according to the sign of the error $(e)$ as follows:

$$
u_{\mathrm{r}}(t)= \begin{cases}h_{+}>0 & e(t)>0 \\ h_{-}<0 & e(t)<0\end{cases}
$$

The relay is called a symmetric relay if $h_{+}=\left|h_{-}\right|$; otherwise, it is called an asymmetric relay. From the relay feedback test, two parameters are normally ob-

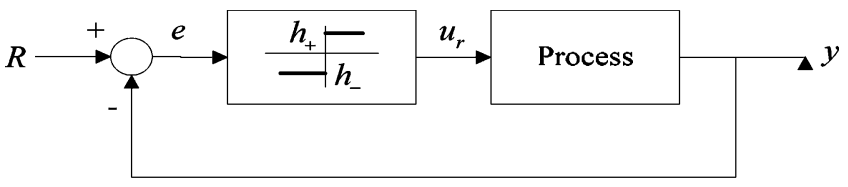

(a)

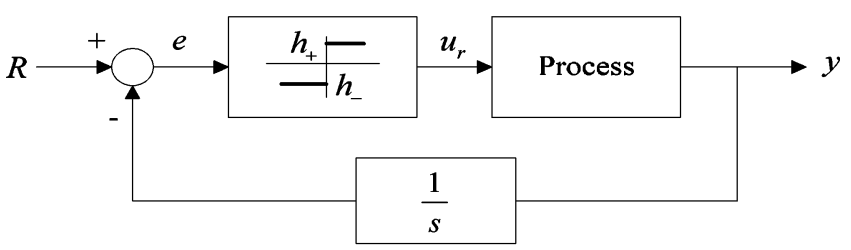

(b)

Figure 2. (a) Relay feedback experiment for structure identification of the nonlinear process and parameter estimation of the Wiener-type process. (b) Relay feedback experiment for parameter estimation of the Hammerstein-type process.

Table 1. Features of the Response in the Symmetric Relay Feedback Test

\begin{tabular}{llll}
\hline & $\begin{array}{l}\text { linear } \\
\text { process }\end{array}$ & $\begin{array}{c}\text { Wiener-type } \\
\text { process }\end{array}$ & $\begin{array}{c}\text { Hammerstein-type } \\
\text { process }\end{array}$ \\
\hline period & $T_{+}=T_{-}$ & $T_{+}=T_{-}$ & $T_{+} \neq T_{-}$ \\
amplitude & $a_{+}=a_{-}$ & $a_{+} \neq a_{-}$ & $a_{+} \neq a_{-}$
\end{tabular}

tained. One is the amplitude of the periodic output curve, and the other is the period. $T_{+}$(or $T_{-}$) and $a_{+}$(or $\left.a_{-}\right)$are the time period and maximum amplitude, respectively, in a constant cycle, during which the system has positive (or negative) output. If the process in this relay feedback loop is a linear one, symmetric output cycles with $T_{+}=T_{-}$and $a_{+}=a_{-}$will be observed. However, with the existence of static nonlinearity in a nonlinear process, the symmetry in the output of the relay and the system disappears. With different sequences of blocks in a nonlinear model, the features of the output cycles differ. Huang et al. ${ }^{15}$ presented a strategy for selecting a feasible model structure based on the observed outputs from a symmetric relay feedback test. Table 1 summarizes the important features of the output curve from linear, Wiener, and Hammerstein systems. On the basis of the results as shown in Table 1, the feasible model structure of the nonlinear dynamic system can be identified. After the model structure has been determined, estimation of the parameters can then proceed.

Although the identification of the model structures is derived from the dynamic behavior of the Wiener and Hammerstein processes, it may apply to WienerHammerstein-type or Hammerstein-Wiener-type processes within a little more conservative range.

\section{Parameter Estimations of Nonlinear Processes}

The proposed procedures for parameter estimations of nonlinear processes apply to a class of processes with monotonic and static nonlinear function. They consist of two steps. Because this proposed method will be focused on their application to control, especially for proportional-integral-derivative (PID; linear or nonlinear) systems, the linear model structure adopted for identification will be confined to transfer functions of FOPDT or SOPDT. First, to identify the static nonlinear function, the relay feedback test of the nonlinear process 

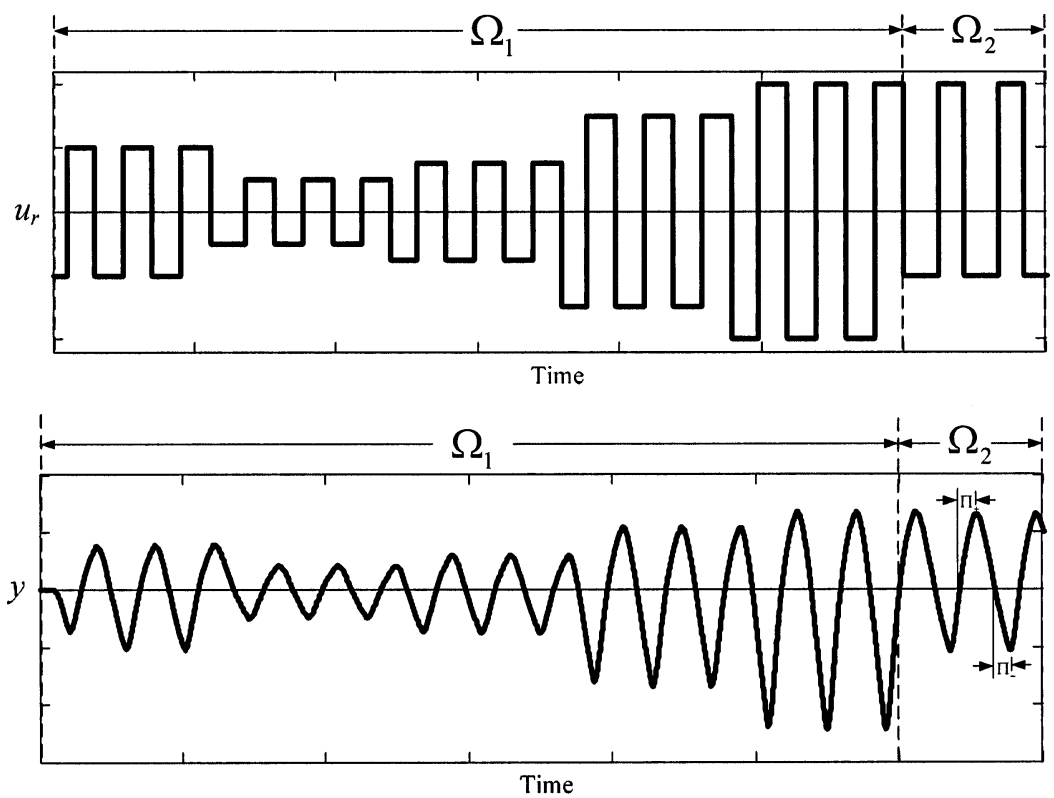

Figure 3. Response of the relay feedback experiment for identification of the Wiener-type process.

is conducted, where the outputs of relay are sequentially assigned to have different magnitudes. Then, a linear model of FOPDT or of SOPDT will be identified by making use of frequency analysis of the resulting cycling responses. One of the particular reasons for using the frequency-domain method is that it estimates the ultimate frequency better than other time-domain methods, especially when a low-order model is the goal. The details of identification procedures for these two types of models will be described in the following.

3.1. Identification Procedures for the WienerType Model. If a Wiener-type model is identified to be appropriate for a nonlinear process, the remaining work is to estimate the parameters in the model. Toward this purpose, the relay feedback experiment is conducted in two periods of time. In the first period of time, designated as $\Omega_{1}$ in Figure 3 , a sequence of different magnitude levels of symmetric relay outputs are used. Each level of magnitude is applied over two or three or more cycles and is switched to another level and so on. At this stage, the maximum and minimum values of the constant cycles for each different level of relay output are recorded. In the second period of time, designated as $\Omega_{2}$, the relay becomes slightly asymmetric and the system outputs in one constant cycle are collected. The entire input and output responses of such an experiment are as shown in Figure 3.

After completion of the experiment, the static nonlinear function is estimated by a nonlinear curve-fitting procedure using data generated from stage $\Omega_{1}$. When the static nonlinearity is finished, the internal variable $v(t)$ is reconstructed through an inverse function of the obtained nonlinear function. Also, a linear model of FOPDT or SOPDT is identified using $u(t)$ and $v(t)$ in the time period of $\Omega_{2}$ as the input and output of the linear subsystem, respectively. The details of the identification procedures are given as follows.

3.1.1. Identification of a Static Nonlinear Function. Assume that $h_{+, i}$ and $h_{-, i}$ are the relay outputs, which are symmetric $\left(h_{+, i}=\left|h_{-, i}\right|=h_{i}\right)$ in the period of $\Omega_{1}$. For the Wiener-type process, the relay shifts synchronically with $v(t)$, which is proportional in magnitude to the magnitude of the relay output being applied when a stable limit cycle is achieved. Thus, the following results can be found in each designed magnitude of the relay signals:

$$
\begin{array}{lll}
\frac{h_{+, i}}{h_{+, 1}}=\frac{v_{+, i}}{v_{+, 1}} & \text { or } & \frac{h_{+, i}}{h_{1}}=\frac{v_{+, i}}{v_{1}} \\
\frac{h_{-, i}}{h_{-, 1}}=\frac{v_{-, i}}{v_{-, 1}} & \text { or } & \frac{h_{-, i}}{h_{1}}=\frac{v_{-, i}}{v_{1}}
\end{array}
$$

where $v_{+, i}$ and $v_{-, i}$ represent the maximum and minimum of $v(t)$, respectively, in one constant cycle. Notice that $v_{+, i}=\left|v_{-, i}\right|=v_{i}$.

It is important to note that, in the block-oriented nonlinear models such as the Wiener one, any two $\left[G_{\mathrm{p}^{-}}\right.$ $(s) / \alpha, N(\alpha)$ ] pairs with different values of $\alpha(\alpha \neq 0)$ are essentially equivalent. To make the identification consistent and unique, when a proper $\alpha$ is chosen, the value of $v_{1}$ can be taken as 1 . With this assumption and the relations in eq 2 , although being unmeasurable, the maximum and minimum values of $v(t)$ can be implied by the relay magnitude being applied.

The static nonlinearity $[N(\cdot)]$ then maps the internal variables $v_{+, i}$ and $v_{-, i}$ to $y_{+, i}$ and $y_{-, i}$, respectively, where $y_{+, i}$ and $y_{-, i}$ are the maximum and minimum values of system output $y(t)$. That is, for each designed relay signal, we have

$$
\begin{aligned}
& y_{+, i}=N\left(v_{+, i}\right)=N\left(h_{+, i} / h_{1}\right) \\
& y_{-, i}=N\left(v_{-, i}\right)=N\left(h_{-, i} / h_{1}\right)
\end{aligned}
$$

As a result, for each relay magnitude (i.e., for each $i$ ), two points, $\left(h_{+, i} / h_{1}, y_{+, i}\right)$ and $\left(h_{-, i} / h_{1}, y_{-, i}\right)$, on the $N(\cdot)$ curve can be obtained.

After all of the data sets of $\left(h_{+, i} / h_{1}, y_{+, i}\right)$ and $\left(h_{-, i} / h_{1}\right.$, $\left.y_{-, i}\right), i=1,2, \ldots, I$, are collected, the nonlinear static function is identified to correlate these data sets by linear regression. Let $\boldsymbol{\theta}$ be the parameter vector of the nonlinear static function to be determined, i.e., $y=$ $\hat{N}(v ; \boldsymbol{\theta})$. Usually, this function is a polynomial and $\boldsymbol{\theta}$ consists of its coefficients of different orders. Thus, the 
identification problem for the static nonlinearity is formulated as the following curve-fitting problem:

$$
\begin{aligned}
& \boldsymbol{\theta}^{*}= \\
& \arg \min _{\boldsymbol{\theta}} \sum_{i}\left\{\left[\hat{N}\left(\frac{h_{+, i}}{h_{1}} ; \boldsymbol{\theta}\right)-y_{+, i}\right]^{2}+\left[\hat{N}\left(\frac{h_{-, i}}{h_{1}} ; \boldsymbol{\theta}\right)-y_{-, i}\right]^{2}\right\}
\end{aligned}
$$

Notice that a standard least-squares software routine can be used to solve this above problem. At the same time, the inverse function of the static nonlinearity, $\hat{N}^{-1}(\cdot)$, can also be found using the same data set in reverse order. This inverse function will be used in the next step to identify the dynamic linear subsystem. More importantly, the inverse function is very useful for the nonlinear controller design.

3.1.2. Identification of a Dynamic Linear Subsystem. Because the inverse function of the static nonlinearity of the process has been identified, the unmeasurable internal signal can be reconstructed from $\hat{v}(t)=\hat{N}^{-1}[y(t)]$. As a result, by taking $\hat{v}(t)$ as the system output, identification of a linear subsystem in a Wiener model is the same as that of a linear system in a relay feedback test.

The data collected in the period of $\Omega_{2}$, where the relay output is asymmetric, are used in this step. The asymmetric relay employed has two main purposes. One is to compute the steady-state gain of the linear subsystem, and the other one is to classify the model structure of this linear subsystem.

When the system at stage $\Omega_{2}$ exhibits constant cycles with period $P_{\mathrm{r}}$, from the dc components of the input and output of the linear subsystem, i.e., $u_{\mathrm{r}}(t)$ and $\hat{v}(t)$, the steady-state gain of $G_{\mathrm{p}}(s)$ can be computed as

$$
K=\frac{\int_{t}^{t+P_{\mathrm{r}}} \hat{v}(t) \mathrm{d} t}{\int_{t}^{t+P_{\mathrm{r}}} u_{\mathrm{r}}(t) \mathrm{d} t}
$$

Also, the frequency responses of $G_{\mathrm{p}}(s)$ at certain nonzero frequencies can be estimated from the coefficients of Fourier series expansion of $u_{\mathrm{r}}(t)$ and $\hat{v}(t)$ as

$$
G_{\mathrm{p}}\left(j n \omega_{\mathrm{r}}\right)=\frac{\int_{t}^{t+P_{\mathrm{r}}} \hat{v}(t) \mathrm{e}^{-j n \omega_{\mathrm{r}} t} \mathrm{~d} t}{\int_{t}^{t+P_{\mathrm{r}}} u_{\mathrm{r}}(t) \mathrm{e}^{-j n \omega_{\mathrm{r}} t} \mathrm{~d} t} \quad \text { for } n=1,2,3, \ldots
$$

where $\omega_{\mathrm{r}}=2 \pi / P_{\mathrm{r}}$. So far, the steady-state gain and frequency response, at the frequency $n \omega_{\mathrm{r}}$, of $G_{\mathrm{p}}(s)$ have been estimated. Next, a transfer function model for the linear subsystem is to be identified. The models considered for this identification are confined to being of FOPDT or SOPDT. This is because these two types of models resemble most dynamic behaviors of real processes. Model structures between these two are determined along with the estimation of the parameters in that model being considered. The procedures of the identification of such a linear model are described as follows.

(i) Model Structure Identification. For a truly first-order process, its dead time is equal to the time period from $\hat{v}=0$ to when $\hat{v}$ reaches a peak (designated as $\Pi_{+}$) or that from $\hat{v}=0$ to when $\hat{v}$ reaches a valley (designated as $\Pi_{-}$), in an asymmetric relay feedback test. Otherwise, for a higher-order process, $\Pi_{+}$will not be equal to $\Pi_{-}$. Accordingly, a simple criterion to validate if a FOPDT model is adequate for representing the linear subsystem is to check if $\Pi_{+} / \Pi_{-}$is close enough to 1 . This criterion can be written as

$$
\Delta=\left|1-\frac{\Pi_{+}}{\Pi_{-}}\right| \leq \epsilon
$$

where $\epsilon$ is an arbitrarily small value as the tolerance for estimation error. When a proper value is given to $\epsilon$, eq 7 is used to justify the adequacy of a FOPDT model. If eq 7 is not satisfied, the linear subsystem would be identified as a SOPDT model.

(ii) Estimation of the FOPDT Model. Assume that the unknown $G_{\mathrm{p}}(s)$ is represented as the following FOPDT model:

$$
\hat{G}_{\mathrm{p}}(s)=\frac{K \mathrm{e}^{-d s}}{\tau s+1}
$$

Using the magnitude of $G_{\mathrm{p}}\left(j \omega_{\mathrm{r}}\right)$, the time constant of the process can be directly calculated as follows:

$$
\tau=\sqrt{\frac{\left(\frac{K}{\mid G_{\mathrm{p}}\left(j \omega_{\mathrm{r}}\right)}\right)^{2}-1}{\omega_{\mathrm{r}}^{2}}}
$$

After $\tau$ has been obtained, the dead time of the process is then estimated by taking the average value as

$$
d=\frac{1}{3}\left[\Pi_{+}+\Pi_{-}+\frac{\arctan \left(-\omega_{\mathrm{r}} \tau\right)-\angle G_{\mathrm{p}}\left(j \omega_{\mathrm{r}}\right)}{\omega_{\mathrm{r}}}\right]
$$

(iii) Estimation of the SOPDT Model. If $G_{\mathrm{p}}(s)$ is found suitable to be characterized by a SOPDT model, then

$$
\hat{G}_{\mathrm{p}}(s)=\frac{K \mathrm{e}^{-d s}}{a s^{2}+b s+1}
$$

The parameters $a$ and $b$ are estimated to minimize the error between $\left|\hat{G}_{\mathrm{p}}\left(j n \omega_{\mathrm{r}}\right)\right|$ and the calculated $\left|G_{\mathrm{p}}\left(j n \omega_{\mathrm{r}}\right)\right|$ from eq 6 , for $n=1$ to some specified value $n_{\mathrm{c}}$. This optimization problem can be simply solved by a leastsquares method, and the values of $a$ and $b$ can be directly estimated from the following equation:

$$
\begin{array}{r}
a^{2}\left|G_{\mathrm{p}}\left(j n \omega_{\mathrm{r}}\right)\right|^{2}\left(n \omega_{\mathrm{r}}\right)^{4}+\left(b^{2}-2 a\right)\left|G_{\mathrm{p}}\left(j n \omega_{\mathrm{r}}\right)\right|^{2}\left(n \omega_{\mathrm{r}}\right)^{2}= \\
K^{2}-\left|G_{\mathrm{p}}\left(j n \omega_{\mathrm{r}}\right)\right|^{2} \quad n=1,2, \ldots, n_{\mathrm{c}}
\end{array}
$$

Once the values of $a$ and $b$ are found, the dead time of the process is estimated as the mean of values obtained from the argument relation at each frequency, i.e.

$$
d=\frac{1}{n_{\mathrm{c} n=1}} \sum_{\mathrm{c}}^{n_{\mathrm{c}}}\left[\frac{\arctan \left(\frac{-b n \omega_{\mathrm{r}}}{1-a n \omega_{\mathrm{r}}^{2}}\right)-\angle G_{\mathrm{p}}\left(j n \omega_{\mathrm{r}}\right)}{n \omega_{\mathrm{r}}}\right]
$$

3.2. Identification Procedures for the Hammerstein-Type Model. For a Hammerstein-type process in a feedback loop that has a symmetric relay controller, the internal signal to the linear subsystem becomes asymmetric because of the static nonlinearity block. In 

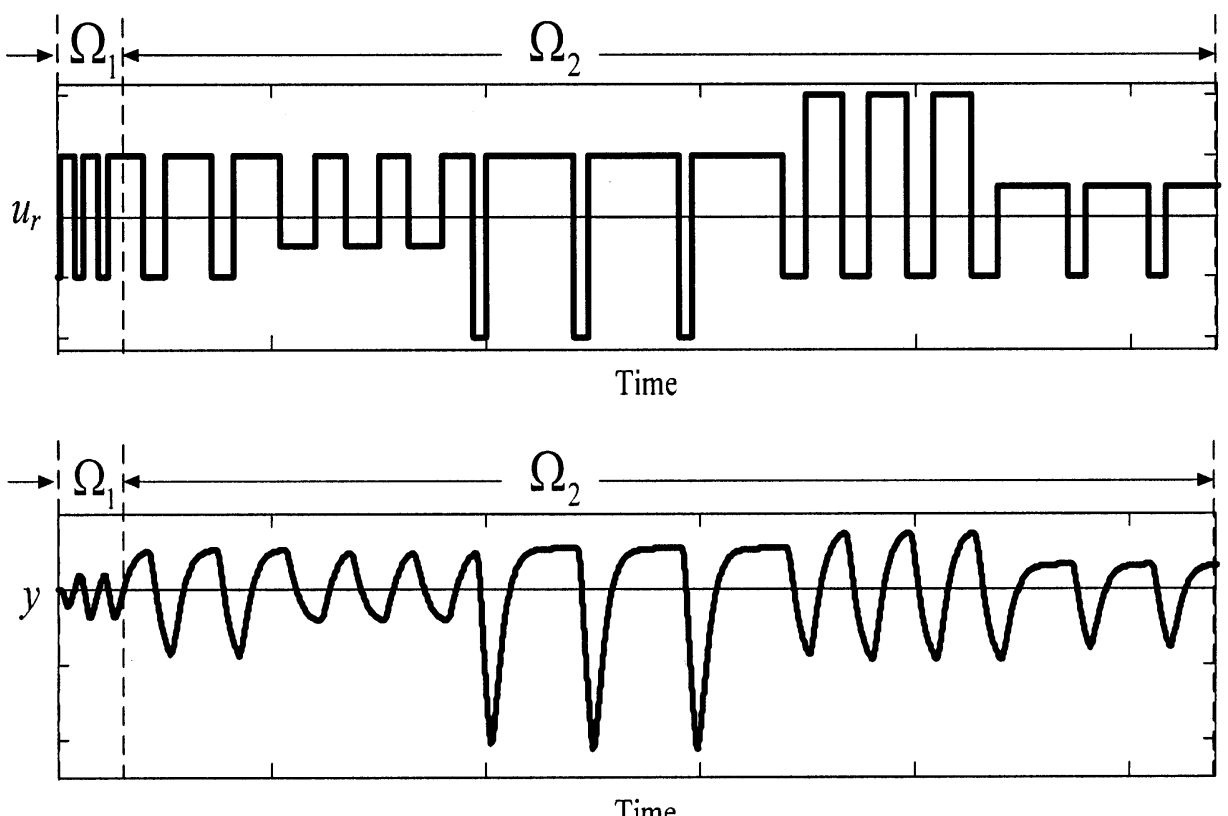

Time

Figure 4. Response of the relay feedback experiment for identification of the Hammerstein-type process.

other words, although having a symmetric input to the Hammerstein process, the linear subsystem is essentially activated by the asymmetric relay signal. Therefore, the output of the linear subsystem will not be symmetric with respect to the setpoint, and the following result will be found for a linear subsystem:

$$
\frac{v_{+}}{v_{-}} \neq \frac{y_{+}}{y_{-}}
$$

where $v_{+}\left[=N\left(h_{+}\right)\right]$and $v_{-}\left[=N\left(h_{-}\right)\right]$represent the values of output from the nonlinear block driven by the relay.

The key to identifying the static nonlinearity in a Hammerstein model is to have several points on the $N($. ) curve. To attain this, the following lemma ${ }^{9}$ will be useful.

Lemma 3.1. If the relay feedback system of a Hammerstein-type process, as shown in Figure 2a, exhibits a stable limit cycle and the linear element $G_{\mathrm{p}}(s)$ includes an integrator, then the following relation holds:

$$
\frac{v_{+}}{v_{-}}=-\frac{T_{-}}{T_{+}}
$$

where $T_{+}$and $T_{-}$are the time durations in a constant cycle during which $v(t)$ has a value of $v_{+}$and $v_{-}$, respectively.

Accordingly, when the nonlinear process is of the Hammerstein type after the symmetric relay feedback test for model structure identification (designated as stage $\Omega_{1}$ ), an additional integrator is inserted into the feedback path of the relay feedback system, as shown in Figure 2b. In this second stage (designated as stage $\Omega_{2}$ ), the relay will see the linear element as if it includes an integrator so that lemma 3.1 can be applied. Consequently, a designed sequence of relay signals is used to estimate the static nonlinearity of the process. This sequence of relay output (i.e., $h_{+, i}$ and $h_{-, i}$ ) is arranged as follows:

(1) For $i=1, h_{+, 1}=\left|h_{-, 1}\right|=h_{1}$. This relay output is the same as that used in model structure identification.
(2) For $i=2,3, \ldots, I_{1}$, the positive relay output is fixed, $h_{+, i}=h_{+, 1}$, and the negative relay output $h_{-, i}$ is varied for different $i$.

(3) For $i=I_{1}+1, \ldots, I_{1}+I_{2}$, the negative relay output is fixed, $h_{-, i}=h_{-, 1}$, and the positive relay output $h_{+, i}$ is varied for different $i$.

Each magnitude of the relay output is applied over two or three cycles to develop constant cycles, and then the relay output is switched to another magnitude and so on. The entire input and output responses of such an experiment are as shown in Figure 4. The procedures for the identification of nonlinear and linear blocks are given in the following.

3.2.1. Identification of the Static Nonlinear Function. The experimental data generated in stage $\Omega_{2}$ are used for identifying the nonlinear block. Let $v_{+, i}\left[=N\left(h_{+, i}\right)\right]$ and $v_{-, i}\left[=N\left(h_{-, i}\right)\right]$ designate the positive and negative values of $v(t)$ when the relay shifts between $h_{+, i}$ and $h_{-, i}$ in stage $\Omega_{2}$. Moreover, $T_{+, i}$ and $T_{-, i}$ are used to represent the time durations in one cycle, within which $v(t)$ and $u_{\mathrm{r}}(t)$ remain positive and negative values, respectively. From the experimental data and applying lemma 3.1, we have

$$
\begin{gathered}
\frac{v_{-, 1}}{v_{+, 1}}=-\frac{T_{+, 1}}{T_{-, 1}}, \quad i=1 \\
\frac{v_{-, i}}{v_{+, i}}=\frac{v_{-, i}}{v_{+, 1}}=-\frac{T_{+, i}}{T_{-, i}}, \quad i=2,3, \ldots, I_{1} \\
\frac{v_{+, i}}{v_{-, i}}=\frac{v_{+, i}}{v_{-, 1}}=-\frac{T_{-, i}}{T_{+, i}}, \quad i=I_{1}+1, \ldots, I_{1}+I_{2}
\end{gathered}
$$

When eq 16 is substituted into eq 18 , it follows that

$$
\frac{v_{+, i}}{v_{+, 1}}=\frac{T_{-, i}}{T_{+, i}} \frac{T_{+, 1}}{T_{-, 1}}, \quad i=I_{1}+1, \ldots, I_{1}+I_{2}
$$

Again, as has been mentioned, the gains of nonlinear and linear blocks are not unique, and any two $[\alpha N(\cdot)$, $\left.G_{\mathrm{p}}(s) / \alpha\right]$ pairs with different values of $\alpha(\alpha \neq 0)$ are 
essentially equivalent for the Hammerstein-type process. Therefore, the value of $v_{+, 1}$ can be normalized to be 1 , without loss of generality. Consequently, with the relations of eqs 16,17 , and 19 , the unmeasurable signal $v(t)$ can be implied by the time durations between shifts of $u_{\mathrm{r}}(t)$, which is measurable. In this way, several points of $\left(h_{+, i}, v_{+, i}\right), i=1, I_{1}+1, \ldots, I_{1}+I_{2}$, and $\left(h_{-, i}, v_{-, i}\right), i=$ $1,2, \ldots, I_{1}$, on the $N(\cdot)$ curve can be obtained.

With these data sets, the static nonlinearity of the process can be approximated by some nonlinear function. The method is similar to that for the Wiener-type model. Let $\boldsymbol{\theta}$ be the parameter vector of the nonlinear static function to be determined, i.e., $v=\hat{N}(u ; \boldsymbol{\theta})$. The identification problem for the static nonlinearity is formulated as the following curve-fitting problem:

$$
\begin{aligned}
\boldsymbol{\theta}^{*}=\arg \min _{\boldsymbol{\theta}}\left\{\sum_{i}\left[\hat{N}\left(h_{+, i} ; \boldsymbol{\theta}\right)-v_{+, i}\right]^{2}+\right. \\
\left.\sum_{i}\left[\hat{N}\left(h_{-, i} ; \boldsymbol{\theta}\right)-v_{-, i}\right]^{2}\right\}
\end{aligned}
$$

Notice that the inverse function of the static nonlinearity is not required for the following identification of the linear subsystem. However, it can be similarly found from the data sets if it is desired for other purposes.

3.2.2. Identification of the Linear Subsystem. For the identification of the linear subsystem, the data of stage $\Omega_{1}$ are used in this step. The procedures of the identification for the linear subsystem of the Hammerstein process are the same as those described in the Wiener process. In stage $\Omega_{1}$, the relay outputs are $h_{+, 1}$ and $h_{-, 1}$, which are mapped into $v_{+, 1}$ and $v_{-, 1}$, respectively, through the nonlinearity. The values of $v_{+, 1}$ (assumed to be 1) and $v_{-, 1}$ have been found in the previous step, and their durations, $T_{+}$and $T_{-}$, can be measured from $u_{\mathrm{r}}(t)$. The identification of the linear subsystem is rather straightforward. First, the steadystate gain of the linear subsystem is computed as

$$
K=\frac{\int_{t}^{t+P_{\mathrm{r}}} y(t) \mathrm{d} t}{\int_{t}^{t+P_{\mathrm{r}}} \hat{v}(t) \mathrm{d} t}=\frac{\int_{t}^{t+P_{\mathrm{r}}} y(t) \mathrm{d} t}{v_{+, 1} T_{+}+v_{-, 1} T_{-}}=\frac{\int_{t}^{t+P_{\mathrm{r}}} y(t) \mathrm{d} t}{T_{+}+v_{-, 1} T_{-}}
$$

where $P_{\mathrm{r}}\left(=T_{+}+T_{-}\right)$is the oscillation period in stage $\Omega_{1}$. In addition, the frequency responses of $G_{\mathrm{p}}(s)$ can be estimated as

$$
\begin{aligned}
& G_{\mathrm{p}}\left(j n \omega_{\mathrm{r}}\right)= \frac{\int_{t}^{t+P_{\mathrm{r}}} y(t) \mathrm{e}^{-j n \omega_{\mathrm{r}} t} \mathrm{~d} t}{\int_{t}^{t+P_{\mathrm{r}}} \hat{v}(t) \mathrm{e}^{-j n \omega_{\mathrm{r}} t} \mathrm{~d} t}= \\
& \frac{\int_{t}^{t+P_{\mathrm{r}}} y(t) \mathrm{e}^{-j n \omega_{\mathrm{r}} t} \mathrm{~d} t}{\int_{t}^{t+T_{+}} \mathrm{e}^{-j n \omega_{\mathrm{r}} t} \mathrm{~d} t+v_{-, 1} \int_{t+T_{+}}^{t+P_{\mathrm{r}}} \mathrm{e}^{-j n \omega_{\mathrm{r}} t} \mathrm{~d} t} \\
& \text { for } n=1,2,3, \ldots
\end{aligned}
$$

For modeling $G_{\mathrm{p}}(s)$ as an FOPDT or SOPDT model, the procedures are described as follows:

(1) In the beginning, the model structure of the linear subsystem is identified by the simple criterion of eq 7 .

(2) If $G_{\mathrm{p}}(s)$ can be represented by an FOPDT model, compute $\tau$ with eq 9 and estimate $d$ with eq 10 .

(3) If $G_{\mathrm{p}}(s)$ has to be modeled as an SOPDT model, estimate $a$ and $b$ with eq 12 through a least-squares method and estimate $d$ with eq 13 .

\section{Remarks.}

(1) The relay signal is designed before the parameter estimations so that no online adaptation of the relay output is required. Performing the relay feedback test in this way for identification will be more convenient than trial with displacing the relay online.

(2) For the Wiener process, in stage $\Omega_{1}$, the number of data points on the $N(\cdot)$ curve can be obtained is $2 I$ ( $I$ for positive input and $I$ for negative input) if the relay outputs are designed as $I$ different magnitudes. For the Hammerstein process, in stage $\Omega_{2}$, the number of data points on the $N(\cdot)$ curve can be obtained is $I_{1}+I_{2}+1$ $\left(I_{2}+1\right.$ for positive input and $I_{1}$ for negative input) if the relay outputs are designed as $I_{1}+I_{2}$ different magnitudes. With more data points obtained, better accuracy can be achieved. Thus, tradeoff is necessary between the accuracy of identification and the experimental time.

(3) The duration of the experiment depends on the nonlinearity of the static function and the dynamics of the process because it is closely related to the polynomial for representing the nonlinearity. Fortunately, for simple monotonic static functions, a low-order polynomial provides feasibility for curve fitting. Thus, the duration of the experiment can be essentially reduced to the time it takes for a few more constant cycles rather than the time it takes for linear systems.

(4) The magnitude of the relay experiment is another issue. The most important issue is to let the nonlinear variable involved cover the possible operating range so that no extrapolation to the nonlinear static model will be needed for practical implementations.

3.3. Adaptation to Measurement Noises. It is enviable that measured output will accompany some noises. For small noises, the data used for identification can be taken as the average values of the measurements from several constant cycles. Therefore, the results from the proposed identification method would not be too sensitive to weak noises. For strong noises, the measured signals need to be pretreated by a filter to reduce the effect of measurement noises. During the relay feedback experiment, the input to the process consists of only periodic pulses. As a result, the output signals from the process will also be periodic within the same frequency range. Without distortions of the major output signal content during filtering, the wavelet transform is one of the most efficient methods recommended for this purpose. Wavelets are a family of basis functions, well localized in both time and frequency domains. Because of their local character, the representation of a signal in the wavelet domain is sparse and allows signal compression and denoising. The procedures of the signal denoising are (1) decomposition of the signal into different frequency bands, (2) thresholding (elimination of small coefficients), and (3) reconstruction of the signal.

The wavelets used in the following simulation work are the discrete Meyer wavelets. After the measurements are denoised, the identification procedures can then be applied.

\section{Illustrative Examples for Identification and Control}

In the following section, the identification together with the controller design will be demonstrated. Once the model of the block-oriented nonlinear process is 


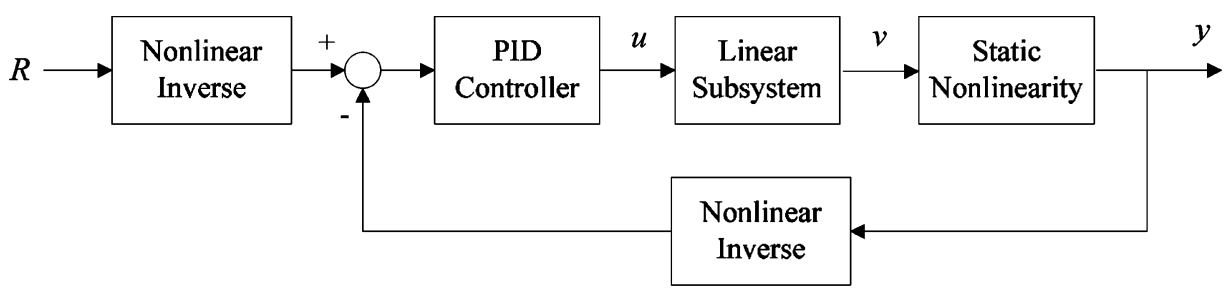

(a)

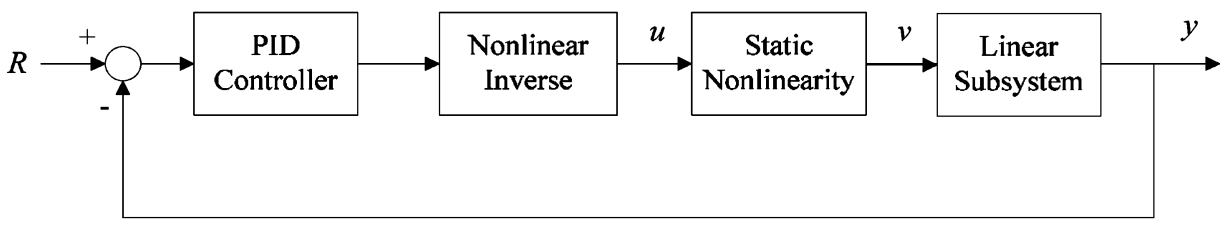

(b)

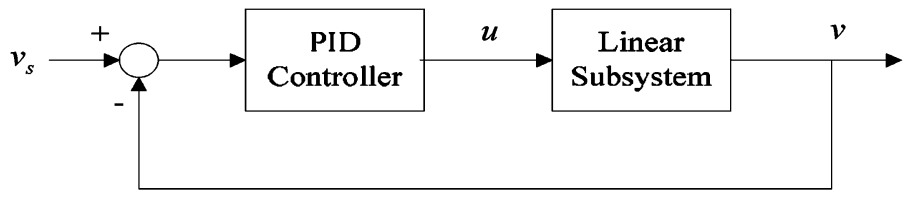

(c)

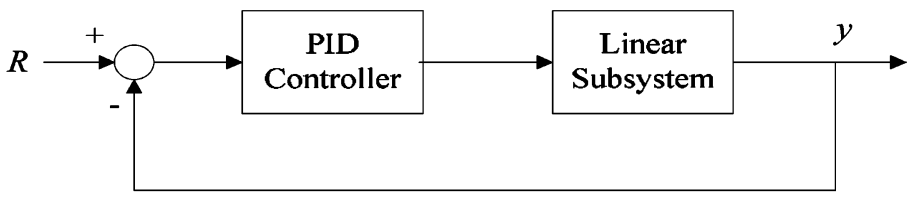

(d)

Figure 5. Control system for nonlinear processes: (a) Wiener process; (b) Hammerstein process. Equivalent control systems; (c) Wiener process; (d) Hammerstein process.

obtained, a control system can be designed accordingly. The control strategies are shown in parts a and b of Figure 5 for the Wiener and Hammerstein processes, respectively. In Figure 5a,b, an inverse of the identified static nonlinear function is imbedded into the control loop to make the system equivalent to a linear loop, as shown in Figure 5c,d. Then, the linear PID controller can be designed, and control performances such as those of linear systems can be achieved. Because the system is equivalent to a linear one, various tuning rules can be applied to the design for the PID controller. For example, to implement the autotuning procedures of Åström and Hägglund, ${ }^{6}$ only the static nonlinear block will be determined, and when the controller is replaced with a relay in the system of parts a or b of Figure 5, the PID controller settings can be easily obtained. On the other hand, if the transfer function for the linear subsystem is also identified, methods of the model-based controller design can be used.

Two example processes are used to demonstrate the proposed identification procedures and control. Example 1 is a Wiener-type process, and example 2 is a Hammerstein-type process.
4.1. Example 1: Wiener Process. Consider a Wienertype process with the following structure:

Linear subsystem

$$
G_{\mathrm{p}}(s)=\frac{1.5 \mathrm{e}^{-s}}{\left(4 s^{2}+2 s+1\right)\left(s^{2}+s+1\right)}
$$

Nonlinear static element

$$
y=\left(1-\mathrm{e}^{-0.25 v}\right) \sqrt{\left|v^{3}\right|}
$$

To show the effect of measurement noise, the output of the process is added with a random number that is of uniform distribution with zero mean and a standard deviation, $\sigma$, of 0.025 . The value of the noise-to-signal variance ratio is about 0.05 , while the relay amplitude is set to 0.5. Because of the presence of measurement noise, the data used for identification are taken as the average values of the measurement from two or three cycles. Figure $6 \mathrm{a}$ (from $t=0$ to 30 ) shows the output response from a symmetric relay $\left(h_{+, 1}=\left|h_{-, 1}\right|=h_{1}=\right.$ 1) feedback experiment. As a result, the time periods 


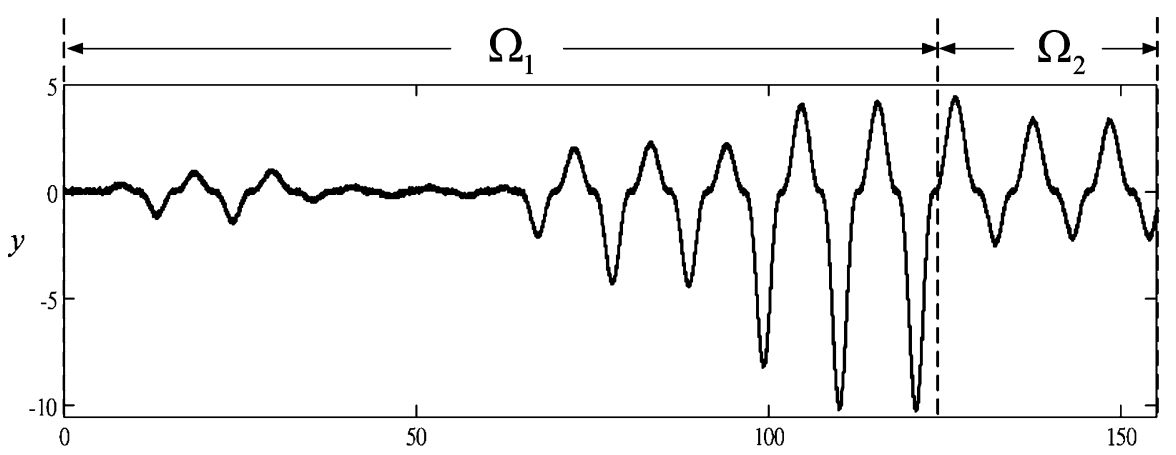

(a)

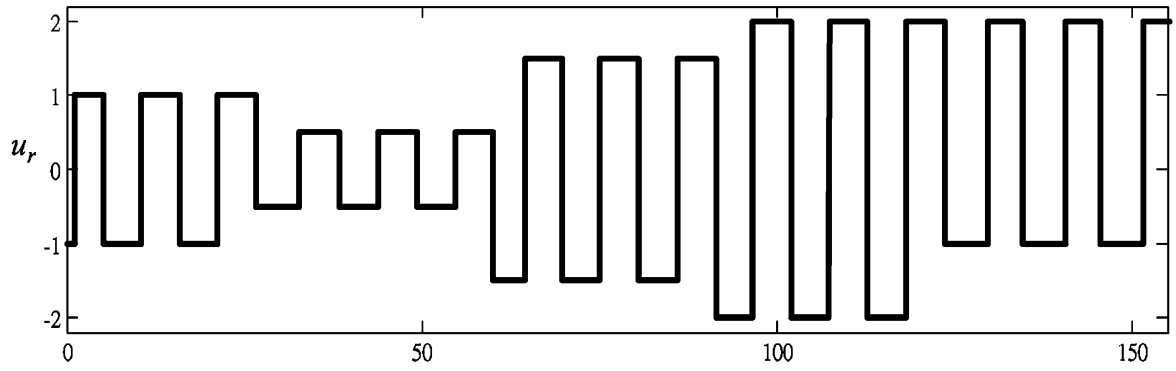

(b)

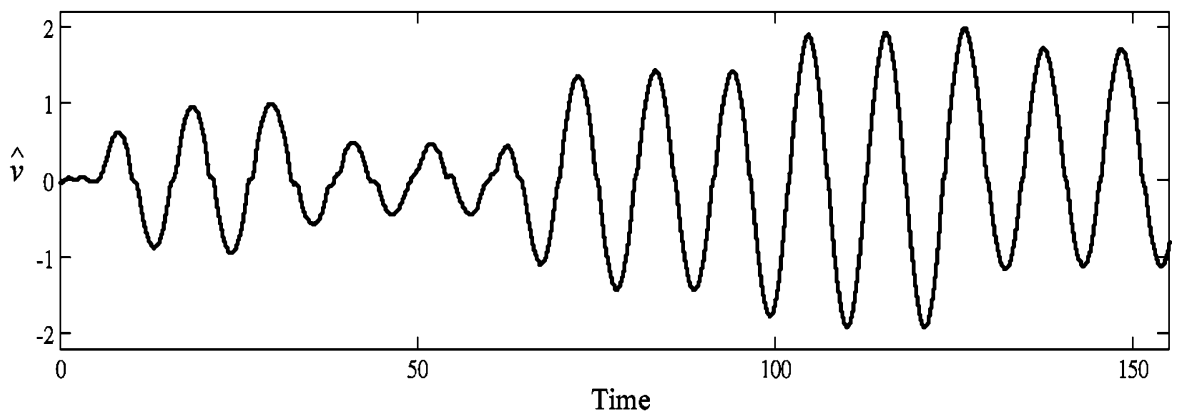

(c)

Figure 6. Relay feedback experiment for example 1: (a) process output $y$; (b) relay output $u_{\mathrm{r}}$; (c) reconstructed internal variable $\hat{v}$.

Table 2. Features of the Responses in Stage $\Omega_{1}$ for Example 1

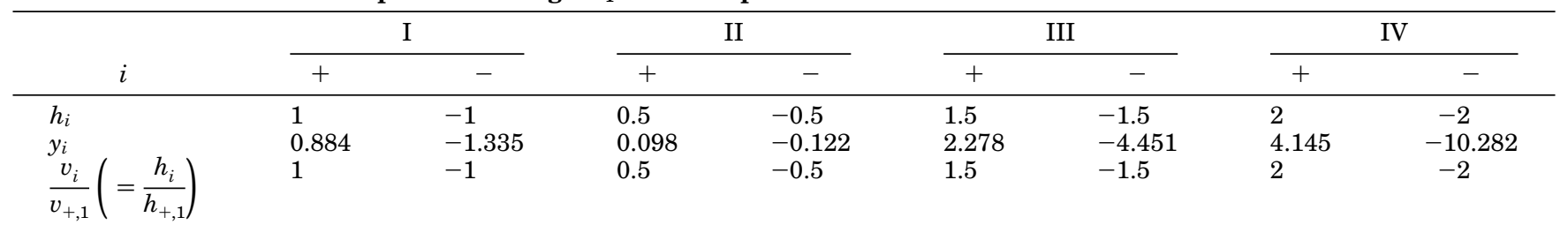

and maximum amplitudes of positive and negative outputs are

$$
T_{+}=5.42 \quad T_{-}=5.40
$$

$$
\left|1-\frac{T_{+}}{T_{-}}\right|=0.004<\epsilon=0.05
$$

$a_{+}=0.884 \quad a_{-}=1.335$

$$
\left|1-\frac{a_{+}}{a_{-}}\right|=0.338 \gg \epsilon=0.05
$$

It is concluded that a Wiener model could fit the process. Because a nonlinear model structure was determined, a sequence of designed relay signals, as shown in Figure $6 \mathrm{~b}$, is used to estimate those parameters of the nonlinear model of the Wiener process. Table 2 summarizes the applied relay magnitudes and features of the process outputs in stage $\Omega_{1}$.

The next step is to identify the static nonlinearity. According to the data of $\left(h_{+, i} / h_{1}, y_{+, i}\right)$ and $\left(h_{-, i} / h_{1}, y_{-, i}\right)$, $i=1, \ldots, 4$, shown in Table 2 , and when an optimization procedure is employed to minimize the objection function of eq 4, the following polynomial is found to approximate the nonlinear static function:

$$
\begin{array}{r}
y=\hat{N}(v)=-0.056 v^{5}-0.171 v^{4}+1.122 v^{3}- \\
0.086 v^{2}+0.010 v
\end{array}
$$




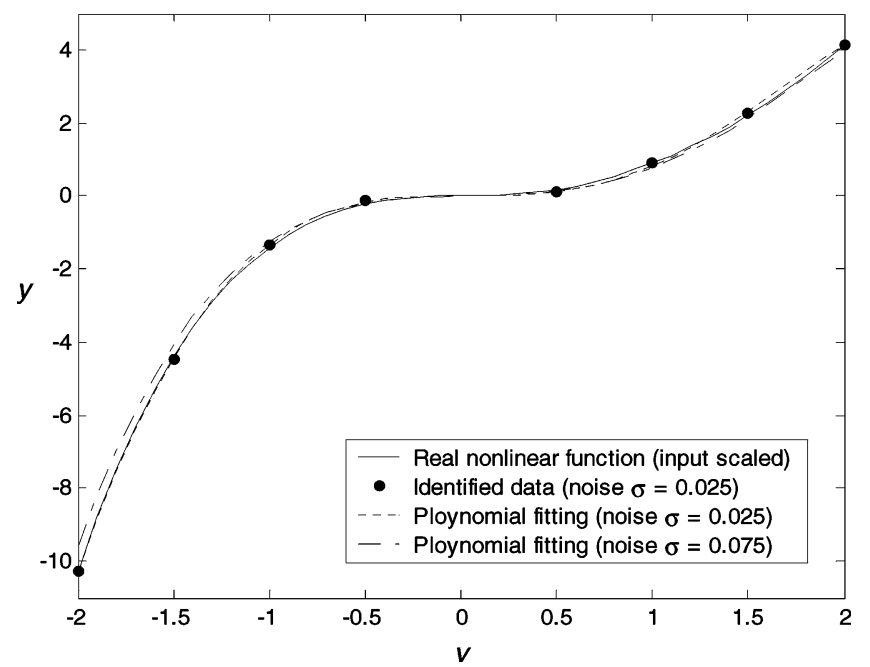

Figure 7. Identification result for the static nonlinear function in example 1.

Figure $6 \mathrm{c}$ shows the internal signal $\hat{v}$ reconstructed by the inverse nonlinear function. Notice that it looks like the typical response of a linear process in a relay feedback loop.

On the basis of the reconstructed $\hat{v}$ in stage $\Omega_{2}$, the steady-state gain and the frequency responses of the linear subsystem are computed by eqs 5 and 6 . Then, a linear transfer function model of FOPDT or SOPDT will be identified. From $\hat{v}$ in stage $\Omega_{2}$, the following result can be found:

$\Pi_{+}=3.08 \quad \Pi_{-}=2.70$

$$
\left|1-\frac{\Pi_{+}}{\Pi_{-}}\right|=0.141 \gg \epsilon=0.05
$$

It is concluded that the SOPDT model could fit the linear subsystem of the process. Consequently, the estimated result is

$$
\hat{G}_{\mathrm{p}}(s)=\frac{0.825 \mathrm{e}^{-2.30 s}}{3.47 s^{2}+1.79 s+1}
$$

Figure 7 shows the result of the identified static nonlinearity where the input of the real nonlinear function is scaled by a factor of $1.818(=1.5 / 0.825)$ to compare it with the identified result. We can see that the proposed identification method provides an excellent nonlinear model. Figure 8 compares the control performance of the proposed nonlinear control strategy and the linear PID controller. The PID parameters are computed using the IMC tuning rule based on a linear dynamic subsystem. The nonlinear controller shows satisfactory responses regardless of the set-point values because it cancels the nonlinearity, whereas the linear PID controller shows acceptable performances for only some set points.

To simulate the presence of stronger noise, a noise of 0.075 standard deviation is added to the output of the process. The noise-to-signal variance ratio is 9 times that of the previous case. To reduce the effect of measurement noise, the output signal is filtered by wavelets before employing the optimization procedure. Accordingly, the identified static nonlinearity is also given in Figure 7, and the result of control is similar to that shown in Figure 8.

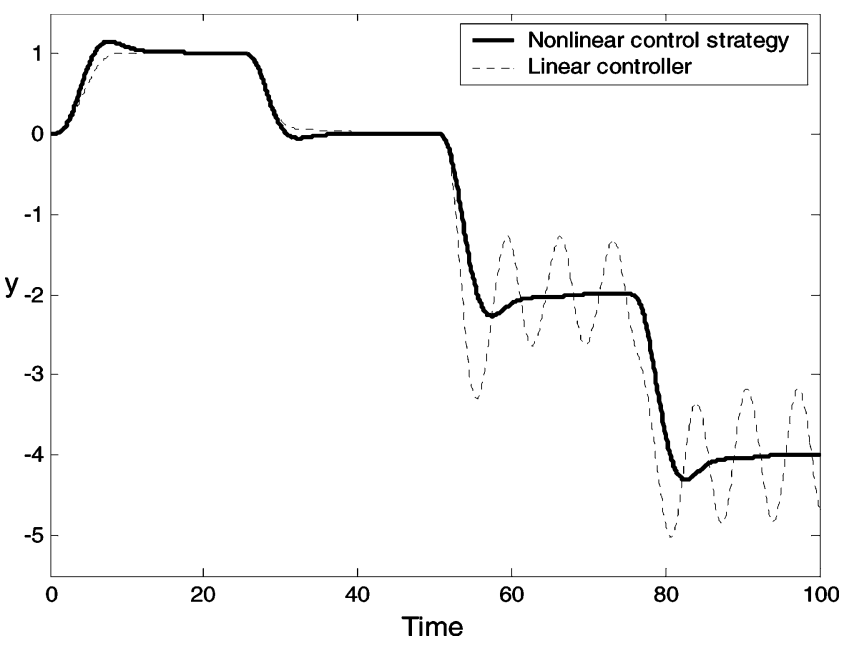

Figure 8. Control performances of example 1.

Table 3. Features of the Responses in Stage $\Omega_{2}$ for Example 2

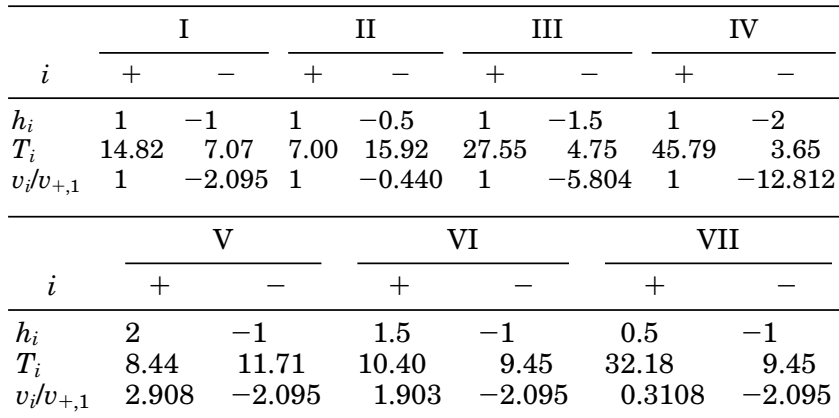

4.2. Example 2: Hammerstein Process. Consider the following Hammerstein-type process:

Nonlinear static element $y=\left(1-\mathrm{e}^{-0.75 u}\right)|u|$

Linear subsystem $G_{\mathrm{p}}(s)=\frac{1}{(s+1)^{5}}$

Similarly, a noise of 0.025 standard deviation is added to the output of the process.

On the basis of the result of the symmetric relay feedback response in stage $\Omega_{1}$, the time periods and maximum amplitudes of positive and negative outputs are

$T_{+}=3.60 \quad T_{-}=5.44$

$$
\left|1-\frac{T_{+}}{T_{-}}\right|=0.339 \gg \epsilon=0.05
$$

$a_{+}=0.255 \quad a_{-}=0.499$

$$
\left|1-\frac{a_{+}}{a_{-}}\right|=0.489 \gg \epsilon=0.05
$$

Obviously, $T_{+} \neq T_{-}$and $a_{+} \neq a_{-}$. It can thus be inferred that a Hammerstein model could fit the process.

To estimate the static nonlinear function, an additional integrator is inserted in the feedback loop (see Figure 2b) and a sequence of designed relay signals are applied to activate the process during stage $\Omega_{2}$. The measured and computed results of stage $\Omega_{2}$ are given in Table 3. Then, on the basis of the data of $\left(h_{+, i}, v_{+, i}\right)$ and $\left(h_{-, i}, v_{-, i}\right)$ shown in Table 3 , the nonlinear static function of this process was estimated by employing an 


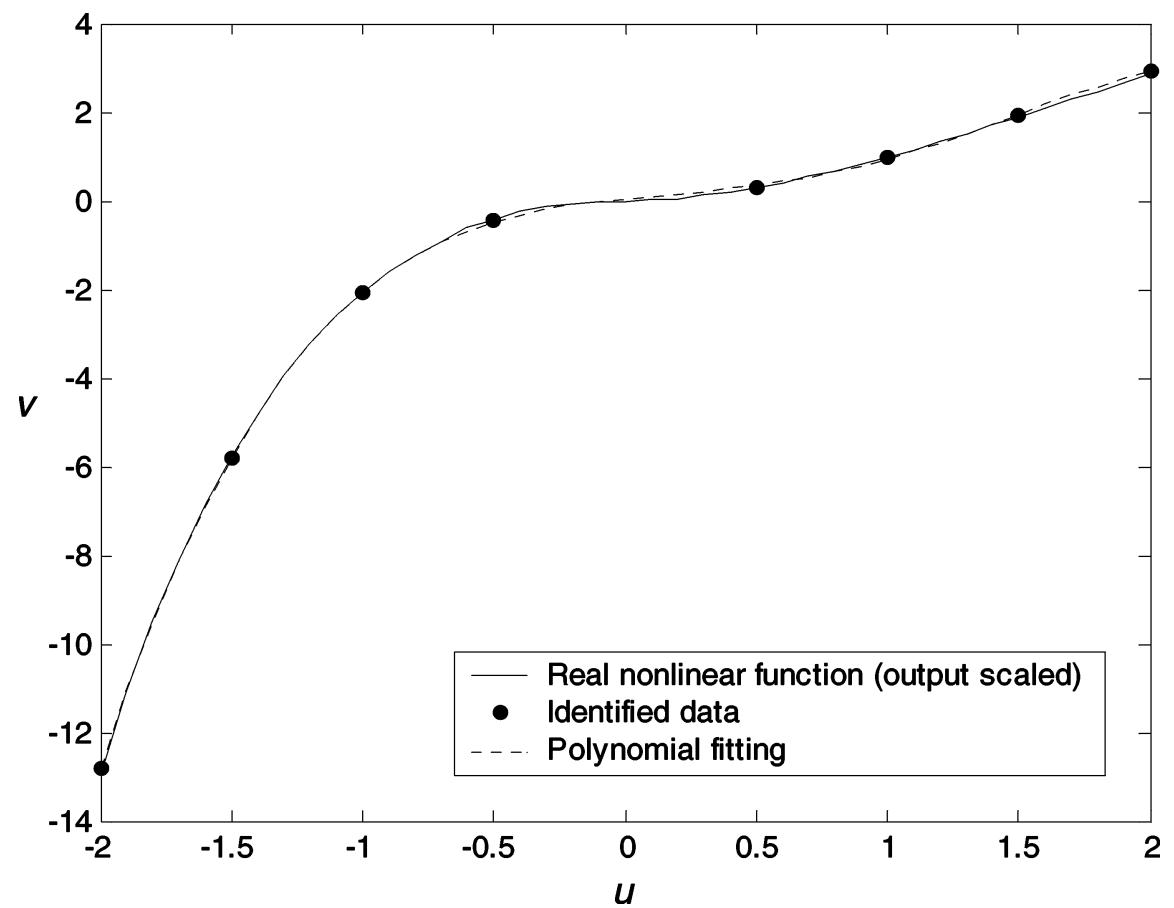

Figure 9. Identification result for the static nonlinear function in example 2.

optimization procedure to minimize the objection function of eq 20 and the result is as the following:

$v=\hat{N}(u)=-0.028 u^{5}-0.214 u^{4}+0.948 u^{3}-$

$$
0.393 u^{2}+0.591 u
$$

where $u \in(-2,2)$.

Now, the outputs of the nonlinear element in stage $\Omega_{1}$ are known, from stage $\Omega_{2}$, as $v_{+, 1}=1$ and $v_{-, 1}=$ -2.095 , so that the steady-state gain and the frequency responses of the linear subsystem are computed by eqs 21 and 22. Furthermore, data measured in stage $\Omega_{1}$ show that

$$
\Pi_{+}=1.84 \quad \Pi_{-}=2.47
$$

$$
\left|1-\frac{\Pi_{+}}{\Pi_{-}}\right|=0.253 \gg \epsilon=0.05
$$

Accordingly, the linear subsystem has to be modeled as SOPDT and the parameters of the model are estimated as follows:

$$
\hat{G}_{\mathrm{p}}(s)=\frac{0.54 \mathrm{e}^{-1.45 s}}{4.24 \mathrm{~s}^{2}+3.43 \mathrm{~s}+1}
$$

Figure 9 shows the result of the identified static nonlinearity where the output of the real nonlinear function is multiplied by $1.852(=1.0 / 0.54)$ to compare it with the identified result. It can be seen that the identified nonlinear function does provide an excellent approximation. Figure 10 compares the control performance of the proposed nonlinear control strategy and the linear PID controller. The nonlinear controller shows almost the same and satisfactory responses regardless of set points because the nonlinearity is removed, whereas the linear PID controller shows a strong dependence on the set-point values.

\section{Conclusions}

In this research, a system of procedures for the identification of Wiener- and Hammerstein-type non-

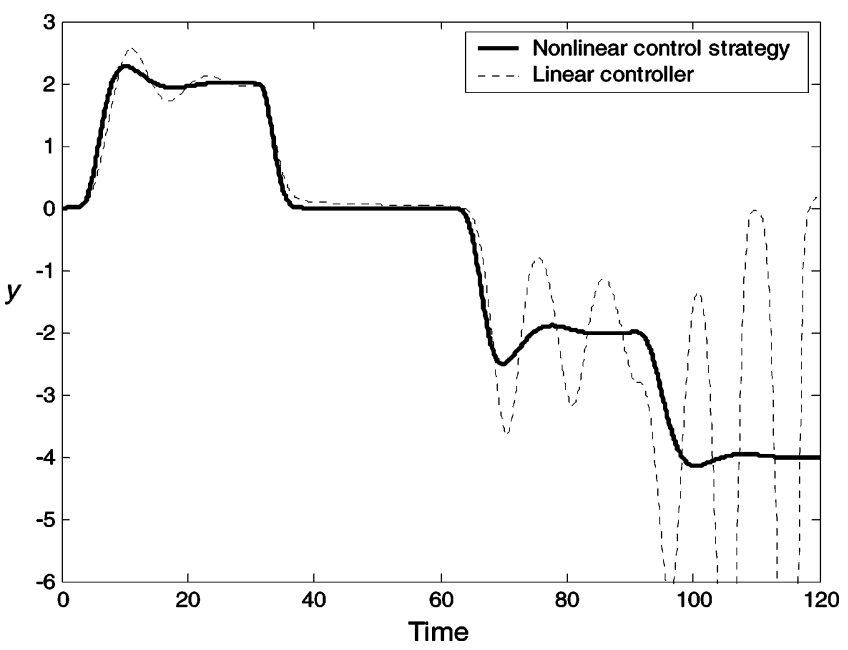

Figure 10. Control performances of example 2.

linear processes have been developed. This method uses a relay feedback test with a sequence of designed relay magnitudes and, for the Hammerstein process, with an integrator inserted in the loop. First, the model structure is selected according to the response in the first few cycles, and then the model parameters of the nonlinear static function and linear dynamic subsystem are estimated in a sequential order. By way of this approach, the identification problems of the nonlinear static function and linear dynamic subsystem are separated and no iterative procedures should be taken. On the basis of the nonlinear model identified, a control system can be designed to realize better control performances than those of a locally linearized controller.

The simulation results show that the proposed identification methods provide satisfactory results, and extension to the multivariable systems will be the next focus of study in the future. 


\section{Literature Cited}

(1) Haber, R.; Unbehauen, H. Structure Identification of Nonlinear Dynamic Systems-A Survey on Input/Output Approaches. Automatica 1990, 26, 651.

(2) Bai, E. W. Frequency Domain Identification of Wiener Models. Automatica 2003, 39, 1521.

(3) Vörös, J. Modeling and Identification of Wiener Systems with Two-Segment Nonlinearities. IEEE Trans. Control Syst. Technol. 2003, 11, 253.

(4) Sung, S. W. System Identification Method for Hammerstein Processes. Ind. Eng. Chem. Res. 2002, 41, 4295.

(5) Bai, E. W. Frequency Domain Identification of Hammerstein Models. IEEE Trans. Autom. Control 2003, 48, 530.

(6) Åström, K. J.; Hägglund, T. Automatic Tuning of Simple Regulators with Specifications on Phase and Amplitude Margins. Automatica 1984, 20, 645.

(7) Huang, H. P.; Lee, M. W.; Tang, Y. T. Identification of Wiener Model Using Relay Feedback Test. J. Chem. Eng. Jpn. 1998, 31, 604 .

(8) Luyben, W. L.; Eskinat, E. Nonlinear Auto-Tuning Identification. Int. J. Control 1994, 59, 595.

(9) Balestrino, A.; Landi, A.; Ould-Zmirli, M.; Sani, L. Automatic Nonlinear Auto-Tuning Method for Hammerstein Modeling of Electrical Drives. IEEE Trans. Ind. Electron. Control Instrum. 2001, 48, 645 .

(10) Sung, S. W.; Lee, J. Modeling and Control of Wiener-Type Processes. Chem. Eng. Sci. 2004, 59, 1515.

(11) Park, H. C.; Koo, D. G.; Youn, J. H.; Lee, J.; Sung, S. W. Relay Feedback Approaches for the Identification of HammersteinType Nonlinear Processes. Ind. Eng. Chem. Res. 2004, 43, 735.

(12) Billings, S. A.; Voon, W. S. F. Structure Detection and Model Validity Tests in the Identification of Nonlinear Systems. IEE Proc. D 1983, 30, 193.

(13) Haber, R. Structure Identification of Quadratic BlockOriented Models Based on Estimated Volterra Kernels. Int. J. Syst. Sci. 1989, 20, 1355.

(14) Menold, P. H.; Allgöwer, F.; Pearson, P. K. Nonlinear Structure Identification of Chemical Processes. Comput. Chem. Eng. 1997, 21, S137.

(15) Huang, H. P.; Lee, M. W.; Tsai, C. Y. Structure Identification for Block-Oriented Nonlinear Models Using Relay Feedback Tests. J. Chem. Eng. Jpn. 2001, 34, 748.

Received for review June 14, 2004 Revised manuscript received January 17, 2005 Accepted January 25, 2005

IE0494840 